\title{
Relación entre el conocimiento y la práctica del autocuidado e hipertensión arterial en los adultos mayores de 35 años del distrito misionero de Chaclacayo de la Iglesia Adventista del Séptimo Día, 2011.
}

\author{
Deisy CONDORI MEZA', Eima SÁNCHEZ RIVERA² y Keila MIRANDA LIMACHI ${ }^{3}$
}

\begin{abstract}
RESUMEN
Objetivo: El objetivo del estudio fue determinar la relación entre nivel de conocimiento y práctica de autocuidado de la hipertensión arterial en los adultos mayores de 35 años del distrito misionero de Chaclacayo de la Iglesia Adventista del Séptimo Día, 2011. Metodología: El estudio fue de tipo cuantitativo, diseño no experimental, descriptivo, correlacional - causal de corte transversal en el distrito misionero de Chaclacayo, mediante un muestreo probabilístico, sobre 123 participantes. Resultados: Respecto a las características de la población en estudio el 70\% y $66,6 \%$ de los que presentaron hipertensión estadios 1 y 2 pertenecían al género femenino y masculino; respecto a la edad, el $35 \%$ y $66,7 \%$ de los que presentaron hipertensión estadios 1 y 2 , pertenecían a la edad etaria de 35 a 45 años y 46 a 56 años. Respecto al grado de estudio el $40 \%$ y $66,7 \%$, de los que presentaron hipertensión estadios 1 y 2, pertenecían al nivel superior. En contraste con antecedentes familiares, el 40\% y $66,6 \%$ de los que presentaron hipertensión estadios 1 y 2, mostraban antecedentes familiares. Con respecto al conocimiento sobre la presencia de hipertensión el 55,0\% y el 100\% de los que presentaron hipertensión arterial estadios 1 y 2, tenían conocimiento sobre la existencia de hipertensión arterial. Según la membresía eclesiástica, el 28,6\% y 20\% de los que presentaron hipertensión estadios 1 pertenecían a las iglesias de la Floresta y Chaclacayo; y el 5\% y 10\% de los que presentaron hipertensión 2 pertenecían a las iglesias de Villa Rica y Chaclacayo. Con respecto a la frecuencia de la hipertensión arterial; se encontró que el $35 \%$ (44) mostraron pre-hipertensión y el $18.7 \%$ (23) hipertensión estadios 1 y 2. Según el nivel de conocimientos de la hipertensión arterial, se encontró que el $55 \%$ y $66,7 \%$ de los que presentaron hipertensión estadios 1 y 2 presentaban nivel de conocimientos bueno y excelente. En el nivel de práctica de autocuidado, se encontró que el $75 \%$ y $99,9 \%$ de los que presentaron hipertensión estadios 1 y 2 obtuvieron un nivel de deficiente a regular y de deficiente a bueno. Conclusiones: Existe una relación positiva muy débil entre el conocimiento y la hipertensión, a diferencia de las prácticas de autocuidado con la hipertensión arterial, debido a que existe una relación positiva considerable. Asimismo, en relación entre el nivel de conocimientos y prácticas de autocuidado con la hipertensión arterial muestran que existe una relación positiva débil.
\end{abstract}

Palabras clave: Conocimiento, práctica de autocuidado, presión arterial, hipertensión arterial, buen estilo de vida.

\begin{abstract}
Objective: The objetive of this study was to determine the relationship between the level of knowledge and self-care practices with hypertension in adults older than 35 years of missionary district of Chaclacayo Adventist Church Seventh Day, 2011. Methods: The study was a quantitative non-experimental, descriptive, correlational -causal cross-section in the missionary district of Chaclacayo, probabilistic sampling, resulting in 123 participants. Results: Regarding the characteristics of the study population el $70 \%$ and $66.6 \%$ of those with hypertension stage 1 and 2 belonged to the female and male, for age, $35 \%$ and $66.7 \%$ of those had hypertension stage 1 and 2 belonged to the age group between 35 and 45 and 46 to 56 years. Study regarding the extent of $40 \%$ and $66.7 \%$ of those with hypertension stage 1 and 2 belonged to the higher grade. In contrast to family history $40 \%$ and $66.6 \%$ of those presenting, hypertension stage 1 and 2, had a family history. With regard to knowledge about the presence of hypertension $55.0 \%$ and $100 \%$ of those with hypertension stage 1 and 2, were aware of the presence of hypertension. According to the churches to $28.6 \%$ and $20 \%$ of those with stage 1 hypertensionbelonged to the churches of the Forest and Chaclacayo, and $5 \%$ and $10 \%$ of those withhypertension, 2 belonged to the churches of Villa Rica Chaclacayo. With respect to the frequency of hypertension, found that 35\% (44) had pre-hypertension, 18.7\% (23) hadhypertension stage 1 and 2. Depending on the level of knowledge of hypertension, found that $55 \%$ and $66.7 \%$ of those with hypertension stage 1 and 2 had excelente.En good level and the level of practice with hypertension, found that $75 \%$ and $99.9 \%$ of those with hypertension stage 1 and 2 were low level to regulate and from poor to good. Conclusions: There is a very weak positive relationship between knowledge and hypertension, as opposed to self-care practices with hypertension, because there is asignificant positive relationship. Also, in relation between the level of knowledge and self-care practices with hypertension show that there is a weak positive relationship.
\end{abstract}

Keywords: Knowledge, practice self-care, blood pressure, high blood pressure, good lifestyle.

\footnotetext{
${ }^{1}$ Licenciada en Enfermería, Universidad Peruana Unión

${ }^{2}$ Licenciada en Enfermería, Universidad Peruana Unión

${ }^{3}$ Magíster en Enfermería con mención en Cuidado de la Salud del Adulto, Docente de la Facultad de Ciencias de la Salud de la Universidad Peruana Unión.
} 


\section{INTRODUCCIÓN}

Según la OMS (2010), "La hipertensión arterial es la primera causa de muerte en el mundo, con una prevalencia promedio de $25 \%$, en un aproximado de 1.000 millones de hipertensos, cifra que se incrementará en los próximos 20 años en más de $50 \%$ de promedio". "La presión arterial es la presión que ejerce la sangre contra la pared de las arterias. Esta presión es imprescindible para que circule la sangre por los vasos sanguíneos y aporte el oxígeno y los nutrientes a todos los órganos del cuerpo para que puedan funcionar" (McPhee \& Ganong, 2007). Según Régulo (2006), esta enfermedad es una de los cinco factores de riesgo que produce más muertes en el mundo. Las estadísticas reportan que 6.9 millones de muertes son por enfermedad isquémica, de los cuales el $49 \%$ son atribuibles a la hipertensión arterial, 5.1 millones de muertes se deben a la enfermedad cardiovascular, de las cuales $62 \%$ también son atribuibles a la hipertensión arterial. Cabe mencionar que en el año 2000, la hipertensión arterial, ya era un problema de salud pública de primera importancia, porque entre el 10 y $30 \%$ de la población adulta a nivel mundial la padecía. Asimismo la OMS (2010), estima que para el 2020 se perderá en todo el mundo un $25 \%$ más de años de vida sana, debido a las enfermedades cardiovasculares producidas por la hipertensión arterial y la mayor parte de este aumento corresponderá a los países en desarrollo.

En el Perú, el 24\% de la población es hipertensa, esto equivale a cinco millones de personas, es decir, uno de cada cuatro peruanos es hipertenso (Ruiz, 2005). Asimismo, la prevalencia de hipertensión en el 2008 en la población general fue de $23,7 \%$ (varones $13.4 \%$ y mujeres $10.3 \%$ ). En la Costa, la prevalencia fue de $27.3 \%$, en la Sierra $22.1 \%$ y en la Selva $22.7 \%$. Estas cifras indican que la posibilidad de desarrollar hipertensión fue mayor en la Costa. (Almirall, 2008).

El 60\% de hipertensos en el Perú, desconocen datos importantes acerca de esta enfermedad, como también los factores de riesgo, los niveles de presión arterial, el tratamiento y la prevención (Ruiz, 2005).

La Iglesia Adventista del Séptimo Día (IASD) promueve la práctica de estilo de vida saludable (como son los 8 remedios naturales: buena nutrición, el ejercicio, consumo de agua, la temperancia, el aire puro, la luz del sol, el descanso y la confianza en Dios) a través del mensaje de reforma pro salud que postula el cuidado del cuerpo de un cristiano, por lo que se espera que el creyente desee salvaguardar su salud por la ley natural, desechando los alimentos que destruyen la salud y los hábitos perjudiciales de vida, siendo moderado en el uso de las cosas que sean buenas (JHH, 2006).
Por otro lado, para lograr el progreso en el control de la HTA, se necesita investigaciones adicionales y mejoras en las tres áreas de conocimiento, tratamiento y control de la HTA. Por ese motivo es relevante la promoción y prevención de la salud, que se debe priorizar en la infancia y la adolescencia.

\section{Estilo de vida}

Es la forma de vida que se basa en patrones de comportamiento identificables, determinados por la interacción entre las características personales individuales, las interacciones sociales y las condiciones de vida socioeconómicas y ambientales. La importancia de mantener un buen estilo de vida es lograr estar sanos y evitar enfermedades crónicas como es la hipertensión arterial, para ello se debe considerar algunos puntos importantes, como son: buen hábito alimenticio, el ejercicio, consumir agua pura, temperancia, descanso y confianza en Dios. Asimismo, Castillo y Orea (2006) informan que el control de estrés es un factor importante para tener un estilo de vida saludable.

El tratamiento de la hipertensión arterial se divide en dos: tratamiento farmacológico y el no farmacológico también conocido como modificación del estilo de vida. Éste último es el que ha demostrado la reducción de cifra de la presión arterial, siendo así un complemento de los tratamientos medicamentosos, permitiendo reducir u obviar las necesidades y dosis de los mismos y retardar su instauración, mejorando en definitiva, la calidad de vida de los pacientes tratados. La utilización de estas medidas representa además menor costo y manifiesta menos efectos adversos que el tratamiento farmacológico (Molina, 2009).

\section{Prácticas de autocuidado}

El autocuidado consiste en la práctica de actividades que las personas maduras, o que están madurando, inician y llevan a cabo en determinados períodos de tiempo, por su propia parte con el interés de mantener un funcionamiento vivo y sano, y continuar con el desarrollo personal y el bienestar mediante la satisfacción de requisitos para la regulación funcional y del desarrollo (Marriner \& Raile, 2007).

\section{MATERIAL Y MÉTODOS}

El estudio fue de tipo cuantitativo, diseño no experimental, descriptivo, correlacional - causal de corte transversal en el distrito misionero de Chaclacayo, mediante un muestreo probabilístico. La población de estudio estuvo conformada por un total de miembros de la IASD adultos mayores de 35 años, registrados hasta el 2010 en las diferentes iglesias del distrito misionero de Chaclacayo. La muestra fue probabilística, para ello se utilizó la fórmula finita, resultando 123 participantes. 
El instrumento de recolección de datos fue un cuestionario elaborado por las investigadoras mediante la operacionalización de las variables y su respectiva validación por juicio de expertos. El cuestionario estuvo divido en tres partes: la primera, estuvo compuesta por los datos generales, la segunda estuvo compuesta por ítems que median el conocimiento de la hipertensión arterial y la tercera parte, por ítems que medían las prácticas de autocuidado en los controles, alimentación saludable, prevención del estrés y del sedentarismo.
Para el análisis estadístico se utilizó el software SPSS, 15.0 y los datos fueron analizados por incidencia y frecuencia, llegando así a emplear como método estadístico la r Pearson, que permitió medir la relación de los puntajes de las variables: conocimiento, prácticas de autocuidado e hipertensión arterial, todo ello con el $95 \%$ de confiabilidad y $5 \%$ de error. Para la organización de los datos estadísticos se usaron gráficos y tablas.

\section{RESULTADOS}

\section{Tabla 1}

Características de la población estudiada según clasificación de presión arterial

\begin{tabular}{|c|c|c|c|c|c|c|c|c|}
\hline \multirow[t]{3}{*}{ CARACTERÍSTICAS } & \multicolumn{8}{|c|}{ Presencia de hipertensión arterial } \\
\hline & \multicolumn{2}{|c|}{ Normal } & \multicolumn{2}{|c|}{ Pre hipertenso } & \multicolumn{2}{|c|}{$\begin{array}{l}\text { Hipertensión } \\
\text { estadio } 1\end{array}$} & \multicolumn{2}{|c|}{$\begin{array}{c}\text { Hipertensión } \\
\text { estadio } 2\end{array}$} \\
\hline & $\mathrm{F}$ & $\%$ & $\mathrm{~F}$ & $\%$ & $\mathrm{~F}$ & $\%$ & $\mathrm{~F}$ & $\%$ \\
\hline \multicolumn{9}{|l|}{ Genero } \\
\hline Femenino & 32 & $(57,1)$ & 25 & $(56,8)$ & 14 & $(70,0)$ & 1 & $(33,3)$ \\
\hline Masculino & 24 & $(42,9)$ & 19 & $(43,2)$ & 6 & $(30,0)$ & 2 & $(66,7)$ \\
\hline \multicolumn{9}{|l|}{ Edad } \\
\hline $35-45$ & 25 & $(44,6)$ & 12 & $(27,3)$ & 7 & $(35,0)$ & 0 & $(0,00)$ \\
\hline $46-56$ & 11 & $(19,6)$ & 13 & $(29,5)$ & 2 & $(10,0)$ & 2 & $(66,7)$ \\
\hline $57-67$ & 11 & $(19,6)$ & 11 & $(25,0)$ & 4 & $(20,0)$ & 0 & $(0,00)$ \\
\hline$>67$ & 9 & $(16,1)$ & 8 & $(18,2)$ & 7 & $(35,0)$ & 1 & $(33,3)$ \\
\hline \multicolumn{9}{|l|}{ Grado de estudios } \\
\hline Sin estudios & 3 & $(5,4)$ & 0 & $(0,00)$ & 1 & $(5,0)$ & 0 & $(0,00)$ \\
\hline Primaria & 9 & $(16,1)$ & 9 & $(20,5)$ & 6 & $(30,0)$ & 1 & $(33,3)$ \\
\hline Segundaria & 19 & $(33,9)$ & 17 & $(38,6)$ & 5 & $(25,0)$ & 0 & $(0,00)$ \\
\hline Superior & 25 & $(44,6)$ & 18 & $(40,9)$ & 8 & $(40,0)$ & 2 & $(66,7)$ \\
\hline \multicolumn{9}{|l|}{ Antecedentes familiares } \\
\hline Mamá & 10 & $(17,9)$ & 8 & $(18,2)$ & 4 & $(20,0)$ & 1 & $(33,3)$ \\
\hline Papá & 13 & $(23,2)$ & 10 & $(22,7)$ & 4 & $(20,0)$ & 1 & $(33,3)$ \\
\hline Hermanos & 2 & $(3,6)$ & 1 & $(2,3)$ & 1 & $(5,0)$ & 0 & $(0,00)$ \\
\hline Ninguno & 30 & $(53,6)$ & 22 & $(50,0)$ & 8 & $(40,0)$ & 0 & $(0,00)$ \\
\hline Otros & 1 & $(1,8)$ & 3 & $(6,8)$ & 3 & $(15,0)$ & 1 & $(33,3)$ \\
\hline \multicolumn{9}{|l|}{ Presión arterial } \\
\hline Sí & 1 & $(1,8)$ & 1 & $(2,3)$ & 11 & $(55,0)$ & 3 & $(100,0)$ \\
\hline No & 41 & $(73,2)$ & 21 & $(47,7)$ & 1 & $(5,0)$ & 0 & $(00,0)$ \\
\hline No sabe & 14 & $(25,0)$ & 22 & $(50,0)$ & 8 & $(40,0)$ & 0 & $(00,0)$ \\
\hline \multicolumn{9}{|l|}{ Iglesias } \\
\hline Floresta & 5 & $(23,8)$ & 10 & $(47,6)$ & 6 & $(28,6)$ & 0 & $(0,00)$ \\
\hline Villa Rica & 9 & $(45,0)$ & 8 & $(40,0)$ & 2 & $(10,0)$ & 1 & $(5,0)$ \\
\hline Chaclacayo & 7 & $(35,0)$ & 7 & $(35,0)$ & 4 & $(20,0)$ & 2 & $(10,0)$ \\
\hline Huampani & 10 & $(50,0)$ & 8 & $(40,0)$ & 2 & $(10,0)$ & 0 & $(0,00)$ \\
\hline Cobián & 11 & $(57,1)$ & 6 & $(28,6)$ & 3 & $(14,3)$ & 0 & $(0,00)$ \\
\hline San Bartolomé & 13 & $(61,9)$ & 5 & $(23,8)$ & 3 & $(14,3)$ & 0 & $(0,00)$ \\
\hline
\end{tabular}


En la tabla 1, respecto a las características de la población estudiada, se encuentra que de las personas que presentaron hipertensión estadio 1 el 70\% fueron de género femenino, a diferencia de los que presentaron hipertensión de estadio 2, el 66, 7 \% eran varones; asimismo, de las personas que presentaron pre-hipertensión el 56,8\% pertenecían al género femenino, al igual que las personas que presentaba presión arterial normal $(57,1 \%)$. Con respecto a la edad, se encontró que de las personas que presentaban hipertensión estadio 2 el 66,7 \% pertenecía a las edades de 46 a 56 años, seguido por las personas que presentaban presiones arteriales normales, los cuales el 44,6\% pertenecía a las edades de 35 a 45 años. Asimismo, de los que presentaron hipertensión de estadio 1, el 35\% pertenecía a las edades de 35 a 45 años al igual de los que eran mayores de 67 años; solo un 29,5\% pertenecían a las edades de 46 a 56 años. Referente al grado de estudio, las personas que presentaron hipertensión arterial estadio 2, el 66,7\% era de grado superior, al igual de las personas que presentaban hipertensión estadio 1, de las cuales el $40 \%$ era de grado superior. Asimismo, de las personas que presentaron pre hipertensión el 40,90\% era de grado superior. Respecto a los antecedentes familiares directos, se identificó que de las personas que presentaron hipertensión estadio 2 el 66,6\% manifestó tener como antecedente familiar a la madre y al padre, a diferencia de las personas que presentaron hipertensión arterial estadio 1, de las cuales el $40 \%$ también manifestó presentar como antecedente familiar a la madre o al padre. Asimismo, de las personas que presentaron pre hipertensión el 40,9\% manifestó presentar como antecedente familiar al padre y a la madre. Según el conocimiento de la presencia de la hipertensión arterial, de las personas que presentaron hipertensión arterial estadio 1 y 2, el 55,0\% y el 100\% sí tenían conocimiento sobre la presencia de la hipertensión arterial, a diferencia de las personas que presentaron pre-hipertensión, de los cuales el 50,0\% no sabía que presentaba un índice de hipertensión arterial. Con respecto a las iglesias, se identificó que de las personas que presentaron hipertensión arterial estadio 2 el 10\% pertenecían a la iglesia de Chaclacayo, seguido por la iglesia de Villa Rica con un 5\%. De las personas que presentaron hipertensión estadio 1, el 28,6\% pertenecía a la iglesia de la Floresta y un $20 \%$, la iglesia de Chaclacayo; asimismo, de las personas que presentaban pre-hipertensión el $47,6 \%$ pertenecía a la iglesia de la Floresta.

Tabla 2 Frecuencia de la hipertensión arterial en los adultos mayores de 35 años del
distrito misionero de Chaclacayo

\begin{tabular}{clcc}
\hline & & Frecuencia & Porcentaje \\
\hline Válidos & Normal & 56 & 45,5 \\
& Pre-hipertensión & 44 & 35,8 \\
& Hipertensión estadio 1 & 20 & 16,3 \\
& Hipertensión estadio 2 & 3 & 2,4 \\
& Total & 123 & 100,0 \\
\hline
\end{tabular}

En la tabla 2 se observa que según la clasificación de presión arterial de la OMS, el 35\% (44) de adultos mayores de 35 años presentó pre-hipertensión; asimismo, 18.7\% (23) adultos presentó hipertensión estadio 1 y 2 , y solo un $45.5 \%$, (56) de adultos mayores de 35 años presentó presión arterial normal.

Tabla 3

Nivel de conocimientos según la hipertensión arterial en los adultos mayores de 35 años del distrito misionero de Chaclacayo.

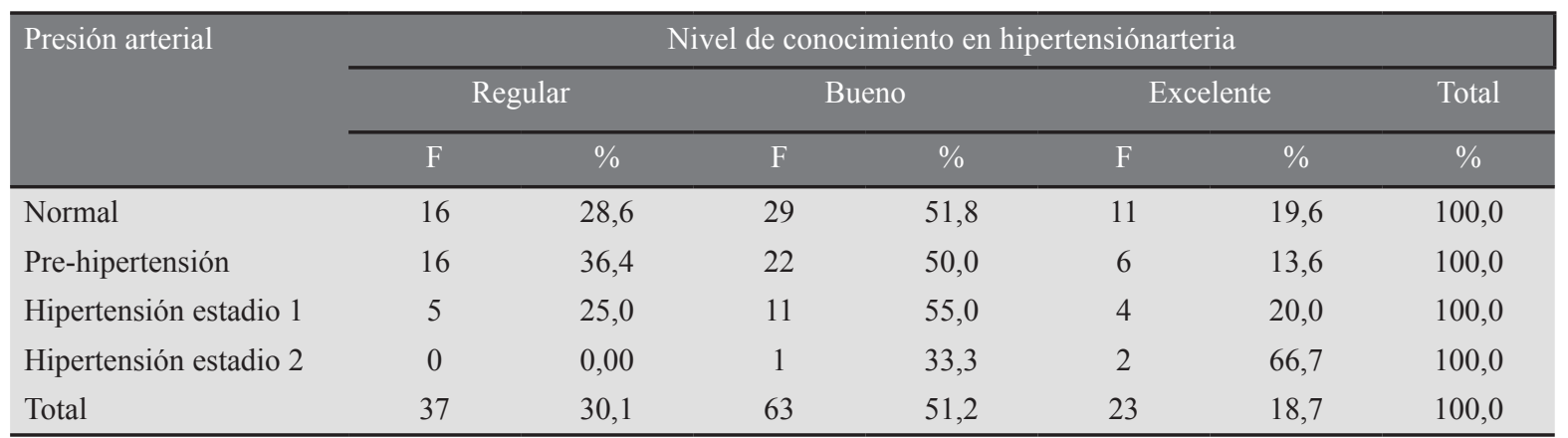


En la tabla 3 se observa respecto al nivel de conocimientos de la hipertensión arterial, las personas que presentaron hipertensión estadio 2, el $66.7 \%$ tuvo conocimientos excelentes; asimismo, las personas que presentaron hipertensión de estadio 1, el 55\% obtuvo un nivel bueno y de los que presentaron presión arterial normal, el 19.6\% tuvo conocimientos excelentes. Lo que indica que las personas de hipertensión estadio 1 ó 2 presentan mejores conocimientos en escala de bueno a excelente.

\section{Tabla 4}

Nivel de práctica de autocuidado según la hipertensión arterial en los adultos mayores de 35 años del distrito misionero de Chaclacayo.

\begin{tabular}{|c|c|c|c|c|c|c|c|c|c|c|c|}
\hline \multirow[t]{3}{*}{ Presión arterial } & \multicolumn{11}{|c|}{ Nivel de práctica de autocuidado en hipertensión arterial } \\
\hline & \multicolumn{2}{|c|}{ Malo } & \multicolumn{2}{|c|}{ Deficiente } & \multicolumn{2}{|c|}{ Regular } & \multicolumn{2}{|c|}{ Bueno } & \multicolumn{2}{|c|}{ Excelente } & \multirow{2}{*}{$\frac{\text { Total }}{\%}$} \\
\hline & $\mathrm{F}$ & $\%$ & $\mathrm{~F}$ & $\%$ & $\mathrm{~F}$ & $\%$ & $\mathrm{~F}$ & $\%$ & $\mathrm{~F}$ & $\%$ & \\
\hline Normal & 0 & 0,00 & 4 & 7,1 & 30 & 53,6 & 18 & 32,1 & 4 & 7,1 & 100,0 \\
\hline Pre-hipertensión & 4 & 9,1 & 15 & 34,1 & 21 & 47,7 & 4 & 9,1 & 0 & 0,00 & 100,0 \\
\hline Hipertensión estadio 1 & 0 & 0,00 & 6 & 30,0 & 9 & 45,0 & 5 & 25,0 & 0 & 0,00 & 100,0 \\
\hline Hipertensión estadio 2 & 0 & 0,00 & 1 & 33,3 & 1 & 33,3 & 1 & 33,3 & 0 & 0,00 & 100,0 \\
\hline Total & 7 & 5,7 & 26 & 21,1 & 61 & 49,6 & 27 & 22,8 & 4 & 3,3 & 100,0 \\
\hline
\end{tabular}

En la tabla 4, respecto al nivel práctica de autocuidado según la hipertensión arterial, los resultados fueron que de las personas con presión normal que presentaron pre hipertensión el 43,2\% presento prácticas de autocuidado entre malo y deficiente; asimismo, de las personas que presentan hipertensión estadio 1, un $75 \%$ presentó un nivel de práctica de deficiente a regular, asimismo, las personas que presentaron hipertensión estadio 2, el 66,6 \% tienen nivel de prácticas deficiente, a regular; y solo el 39,2 \% de las personas que presentó la presión arterial normal obtuvo un nivel de práctica bueno y excelente.

Tabla 5

Análisis estadísticos de los resultados de estudio

\begin{tabular}{|c|c|c|c|c|c|}
\hline Relevancia & $\mathrm{R}$ & SIG & Análisis & $\mathrm{R}^{2}$ & Decisión \\
\hline $\begin{array}{l}\text { Relación entre el nivel de conoci- } \\
\text { mientos con la hipertensión arte- } \\
\text { rial, en el momento de la medición. }\end{array}$ & 0,080 & 0,682 & $\begin{array}{c}\text { Sig }= \\
0,682> \\
0,05\end{array}$ & $\begin{array}{l}0,006 \\
0,6 \%\end{array}$ & $\begin{array}{l}\text { Existe relación } \\
\text { positiva muy } \\
\text { débil. }\end{array}$ \\
\hline $\begin{array}{l}\text { Relación entre el nivel de prácti- } \\
\text { cas de autocuidado con la hiper- } \\
\text { tensión arterial, en el momento de } \\
\text { la medición. }\end{array}$ & 0,730 & 0,001 & $\begin{array}{l}\text { Sig. }= \\
0,001< \\
0,05\end{array}$ & $\begin{array}{c}0,709 \\
70,9 \%\end{array}$ & $\begin{array}{l}\text { Existe relación } \\
\text { positiva } \\
\text { considerable }\end{array}$ \\
\hline $\begin{array}{l}\text { Relación entre el nivel de conoci- } \\
\text { mientos y las prácticas de autocui- } \\
\text { dado con la hipertensión arterial, } \\
\text { en el momento de la medición. }\end{array}$ & 0,344 & 0,001 & $\begin{array}{l}\text { Sig. }= \\
0,001< \\
0,05\end{array}$ & $\begin{array}{c}0,118 \\
11,8 \%\end{array}$ & $\begin{array}{l}\text { Existe relación } \\
\text { pequeña pero } \\
\text { apreciable }\end{array}$ \\
\hline
\end{tabular}

La tabla 5 muestra la relación que tiene el nivel de conocimiento con la hipertensión arterial al momento de medición. La correlación es positiva débil, por lo que presenta un $\mathrm{r}=0,080$; asimismo las variables solo están relacionadas en un $0,6 \%$ presentado por el coeficiente de determinación ( $\mathrm{r} 2=0,006)$; mientras que el sig. $=0,682$ excede la valor de prueba (error) $\mathrm{p}=0.05$ por lo que se concluye que la relación es débil frente a las variables en estudio, concluyendo que se rechaza la hipótesis de investigación por la nula. Por otro lado, la relación que tiene el nivel de práctica de autocuidado con la hipertensión arterial, la relación es positiva considerable, por lo que presenta un $\mathrm{r}=$ 0,709; asimismo las variables solo están relacionadas en un 70,9\% presentado por el coeficiente de determinación 
$(\mathrm{r} 2=0,730)$; asimismo el sig. $=0,001$ excede el valor de prueba (error) $\mathrm{p}=0.05$ por lo que se concluye que la relación es considerable a las variables en estudio, concluyendo que se aprueba la hipótesis de investigación rechazando la nula. Del mismo modo, en la relación que tienen los niveles de conocimiento y prácticas de autocuidado con la hipertensión arterial, la relación muestra que es pequeña pero apreciable, por lo que presenta un $r=0.34$; además, las variables están relacionadas en un $11,8 \%$ presentado por el coeficiente de determinación $(\mathrm{r} 2=0.118)$; asimismo el sig. $=0,001$ excede el valor de prueba (error) $\mathrm{p}=0.05$ por lo que se concluye que la relación es considerable a las variables en estudio, concluyendo que se aprueba la hipótesis de investigación rechazando la nula.

\section{DISCUSIÓN}

De acuerdo a las características de la población estudiada (tabla 1) se encontró que de las personas que presentaron hipertensión estadio 1 el $70 \%$ fue de género femenino, a diferencia de los que presentaron hipertensión de estadio 2 , donde el $66,6 \%$ eran varones. Esto indica que la hipertensión estadio 1 es frecuente en las mujeres de dicha población a diferencia que los varones padecen hipertensión grave, es decir estadio 2. Según Jenkin (2005), menciona que la hipertensión arterial se desarrolla más en los varones que en las mujeres; por otro lado, el género es considerado uno de los factores de riesgo no modificables de la hipertensión arterial, siempre va de la mano con la edad. Asimismo, Saldarriaga (2007), menciona que los varones desarrollan hipertensión entre los 35 y 50 años, mientras que las mujeres son más propensas a padecer hipertensión después de la menopausia.

Con respecto a la edad, se encontró que de las personas que presentaron hipertensión estadio 2 el 66,7 \% pertenecía a las edades de 46 a 56 años, seguido por el 35\% de personas que presentaron hipertensión de estadio 1, que eran de las edades de 35 a 45 años, porcentaje igual al grupo etario de mayores de 67 años. Esto quiere decir que el grupo de edad de 46 a 56 años, es el de mayor riesgo. Así como el género y la edad, los antecedentes familiares también forman parte de uno de los factores de riesgo no modificables. Al respecto, Jenkin (2005) menciona que la hipertensión tiende a ocurrir en familias, lo que se debe principalmente a factores genéticos y, en segundo lugar, a las semejanzas del estilo de vida. Asimismo, en la población estudiada podemos observar que de las personas que presentaron hipertensión estadio 2 , el $66,6 \%$ tenía como antecedentes familiares a su madre o padre.

Por otro lado, como se observa en la tabla 2, según frecuencia de hipertensión arterial, el 35\% (44) de adultos mayores de 35 años presentaron pre-hipertensión, el $18.7 \%$ (23) sumados presentaron hipertensión estadio 1 y 2 , y solo un $45.5 \%$, (56) de los encuestados presentaron presión arterial normal. También se encontró que las personas que mostraron hipertensión arterial estadio 1 y 2 , el $28,6 \%$ y $10 \%$ pertenecían a las iglesias de la Floresta y Chaclacayo, lo que indica que éstas tienen mayor riesgo.
En la tabla 1, sobre los resultados del conocimiento de la presencia de la hipertensión arterial, las personas que presentaron hipertensión arterial estadio 1 y 2 , el $55 \%$ y el $100 \%$ respectivamente conocía su diagnóstico de la presencia de su enfermedad, a diferencia de las personas que presentaron pre-hipertensión, de los cuales el $50 \%$ no sabía que la presentaba, por tanto tienen el riesgo de padecerla más adelante.

Los resultados presentados en la tabla 3, en relación al nivel de conocimientos, muestran que el $18,7 \%$ presentó un nivel excelente; el 51,2\%, un nivel bueno y el $30,1 \%$, un nivel regular. En relación con la hipertensión arterial (tabla 10) se ha identificado que de las personas que presentaron hipertensión de estadio 2 , el $66,7 \%$ revelaron un nivel conocimiento excelente, esto es relativo a los que presentaron hipertensión de estadio 1 , con el $55,0 \%$ presentando un nivel de conocimiento bueno, al igual que las personas que presentan pre-hipertensión con un $50 \%$.

La situación descrita se presentó, probablemente porque los participantes del estudio, en su mayoría, eran profesionales. Según los resultados estadísticos de las personas con hipertensión de estadio 1, el 40\% era profesional de nivel universitario, al igual que las personas con hipertensión de estadio 2. Esto quiere decir que los adultos mayores de 35 años presentan buen conocimiento sobre hipertensión arterial debido a que están capacitados, por asistir a sus controles o por formar parte de algún programa de salud realizado por el Ministerio de Salud relacionados al control de la hipertensión arterial (Sardaña, 2000).

Al respecto, la OMS (2002), menciona que muchas veces toda persona que padece una enfermedad presenta una sensación de ansiedad al ser diagnosticada de hipertensión y/o presentarse síntomas. Las personas recién buscan información en los centros de salud o buscan participar de algún programa de control.

La hipertensión arterial en muchos casos se presenta asintomática y pueden permanecer así por muchos años, pero en otros casos se presenta con una frecuente cefalea sobre todo en las personas diagnosticadas con hipertensión arterial, en contraste con aquellos que presentan la presión elevada y desconocen que son hipertensos (Smeltzer \& Bare, 2005). 
Con respecto a las dimensiones de conocimientos cognitivo y procedimental, existe una diferencia significativa, debido a que los adultos presentaban conocimientos cognitivos de nivel bueno a excelente $(73,17$ $\%$ ), a diferencia de los conocimientos procedimentales, donde los resultados fueron deficiente a bueno $(80,47$ $\%)$. Lo que indica que algunas personas se preocupan más en adquirir conocimientos generales de las enfermedades que en adquirir conocimientos procedimentales, que son los pilares esenciales para la promoción y prevención de la Salud.

Los resultados mencionados indican que los conocimientos no tienen relación con la presencia de una enfermedad, en este caso con la presencia de la hipertensión arterial. Al respecto Rojas (1996), indica que en nuestro país en los últimos años se ha observado que el conocimiento sobre la hipertensión arterial al igual que la incidencia de la hipertensión arterial, es mayor; lo cual indica que los conocimientos no determinan la presencia de una enfermedad.

Por su parte, la OPS y OMS (2000) mencionan que el conocimiento no determina la presencia de la hipertensión arterial, porque el conocimiento no cambia, debido a que éste es un conjunto de informaciones almacenadas mediante experiencias, reglas, interpretaciones y conexiones puestas dentro de un contexto y de una experiencia, mas no determinante en el cambio de las prácticas de autocuidado en la persona (Andreu \& Sieber, 2000).

Existe trabajos similares como el estudio de Saldarriaga (2007), cuya investigación fue titulada "El nivel de conocimiento y práctica de autocuido del paciente hipertenso del Policlínico Juan Rodríguez Lazo con el policlínico Villa María y servicios de transporte de emergencia", Lima; en cuanto a los resultados sobre el conocimiento: el 16,7\% fue excelente, el $31,7 \%$ bueno, el $35 \%$ regular y el $16,7 \%$ deficiente.

Los resultados son presentados en la tabla 4, sobre las prácticas de autocuidado de adultos mayores de 35 años del distrito misionero de Chaclacayo, muestran que las personas con hipertensión de estadio 2 el $66,6 \%$ reveló un nivel de prácticas de autocuidado de deficiente a regular, esto es relativo a los hipertensos de estadio 1, quienes mostraron un $75 \%$ de nivel de deficiente a regular al igual que las personas con prehipertensión, con excepción de los que tienen presión arterial normal, quienes obtuvieron un $85.7 \%$ de nivel regular a bueno respectivamente, lo que indica que las personas con buenas prácticas de autocuidado no presentan hipertensión arterial, a diferencia de las personas que tienen prácticas de autocuidado de deficiente a regular, que sí presentan hipertensión estadio 1 y 2 .
En ese sentido, Velázquez (2011) menciona que la presencia de hipertensión arterial se debe a la alteración de las prácticas de autocuidado o al deficiente estilo de vida que llevan las personas, porque ellas son las que previenen o controlan la presencia de cualquier enfermedad.

Con respecto a las prácticas de autocuidado en controles de salud regular, se encontró que la mayoría de los adultos (94\%), mostró un nivel deficiente a regular y solo el $6 \%$ mostró un nivel excelente. Por otro lado, las personas que presentaron hipertensión de estadio 2 , el $100 \%$ se controlaba regularmente, a diferencia de las personas que evidenciaron hipertensión estadio 1, tuvieron un control malo, al igual de los que tenían pre hipertensión, esto quiere decir que las personas que tienen la enfermedad y no se controlan, pueden llegar a presentar complicaciones y, en el peor de los casos, pueden morir (Sardaña, 2000).

Torreblanca (1997) menciona que estos casos se presentan debido a que hoy en día las personas ya no asisten a sus controles porque en sus resultados presentan una situación positiva, sumado a que en otros casos la espera y el costo de la consulta hacen perder el interés en los controles de salud.

La alimentación también juega un papel principal en la prevención de las enfermedades, porque el cuerpo se forma con el alimento que ingerimos (White, 2007). Asimismo, en el nivel de prácticas de autocuidado en alimentación con hipertensión arterial, el 92,6\% presentó prácticas en alimentación de deficiente a regular, y un $7,4 \%$ presentó un nivel malo. También las personas que presentaron hipertensión de estadio 1 el $15 \%$ presentó malas prácticas en su alimentación y un $65 \%$ presentaron prácticas, en su alimentación, de regular a bueno.

Asimismo, de las personas con hipertensión estadio 2, el 33,3\% presentó prácticas de alimentación malas y un $66,6 \%$, de regular a bueno. Esto quiere decir que a pesar de que las personas tienen la enfermedad, no ejecutan buenas prácticas de autocuidado en la alimentación. Jenkins (2005), menciona que la alimentación es uno de los factores de riesgo modificable de la hipertensión arterial.

Al respecto, Cayetano (2004), menciona que la personas no cuidan su alimentación por el tiempo o el mal hábito que se han adquirido desde niños.

Por otro lado, White (2007) indica que el alimento forma parte de un proceso maravilloso aprovechado en la reconstrucción de las diversas partes del cuerpo, por eso las personas que presentan la enfermedad deberían prestar mayor atención a las costumbres alimenticias. 
Dentro de la dimensión de alimentación se consideró el consumo de sal como factor de riesgo para la HTA, lo que coincide con Diehl \& Ludington (2003), que señala que la HTA es infrecuente en el $80 \%$ en la población donde el consumo de sal no es común a comparación de los lugares donde se usa mucha sal, como en el Japón donde esta enfermedad es mayor.

Pérez, Cordiés, Vásquez y Serrano (2004), recomiendan que se evite consumir alimentos ricos en proteínas de alta calidad como: carne, leche, pescado, mariscos, etc., porque contienen más sodio que la mayoría del resto, a diferencia de los alimentos naturales como son los vegetales y frutas, legumbres, frutas frescas, los cuales contienen cantidades insignificantes de sodio que pueden emplearse libremente.

Se recomienda que la ingestión de sal no sobrepase los 5 gr/por cada 1000 calorías en una persona adulta (Escott \& Mahan, 2009).

Cabe mencionar que la IASD promueve el cuidado del cuerpo mediante la reforma pro salud y un estilo de vida saludable dentro de su mensaje integral. Sin embargo, los resultados en cuanto a la dimensión de la alimentación con práctica de autocuidado, demuestran que las personas con hipertensión arterial estadio 1 y 2 , no mostraban buenas prácticas de alimentación. Esto a pesar de que los adventistas tienen mayor estímulo a un estilo de vida saludable por el estudio sistemático e interpretación bíblica con la que cuentan, sumado a los escritos de Elena White que hablan ampliamente sobre temas del cuidado de la salud.

En cuanto a las prácticas de autocuidado en prevención del estrés y del sedentarismo, encontramos una gran diferencia, porque el 92,6\% mostró prácticas de regular a excelente y solo un $7 \%$ presentó malas prácticas, esto quiere decir que las personas de dicha población tienen buenas prácticas de estrés y sedentarismo.

En este contexto, Rivera (2006) en su estudio titulado "Capacidad de agencia de autocuidado en personas con hipertensión arterial hospitalizadas en una Clínica de Bogotá, Colombia”, encontró que el 53\% de las personas hospitalizadas con hipertensión arterial tuvo deficiente capacidad de agencia para el autocuidado, en tanto que el $47 \%$ presentó suficiente capacidad de agencia para el autocuidado.

Hoy en día las prácticas de autocuidado no se tienen muy en cuenta. En el Perú la incidencia de la hipertensión arterial ha ido en aumento. El año 2005 la incidencia de la hipertensión arterial incrementó un $24 \%$ (cinco millones de personas), en el 2008 la población hipertensa fue de $23,7 \%$ (varones $13.4 \%$ y mujeres
$10.3 \%$ ). En la Costa, la prevalencia fue de $27.3 \%$, en la Sierra 22.1\% y en la Selva 22.7\% (Almirall, 2008).

A pesar de todo el conocimiento que presentan los adultos mayores de 35 años del distrito misionero de Chaclacayo, la prevalencia de hipertensión arterial es alarmante y esto se debe a sus pocas prácticas de autocuidado, como también al índice de antecedentes familiares con hipertensión arterial, lo cual también indica la presencia o la predisposición de hipertensión en los adultos mayores de 35 años del distrito misionero de Chaclacayo.

En conclusión, los resultados muestran que la correlación entre la presión arterial y las prácticas de autocuidado preventivas de la hipertensión arterial en el control, la alimentación saludable y la prevención del estrés y sedentarismo es alta con un $\mathrm{r}=0.730$. Esto significa que la relación entre las variables es considerable; por otro lado, el coeficiente de determinación ( $\mathrm{r} 2=0.709)$ representa que las variables se relacionan en un porcentaje de $70.9 \%$, concluyendo en rechazar la hipótesis nula a favor de la de investigación.

Como se ha descrito, el conocimiento es un factor importante para el ser humano ya que se puede adquirir por hechos, experiencias y datos, pero esto no cambia el hecho de que presente la enfermedad de la hipertensión arterial. A diferencia del conocimiento, las prácticas de autocuidado sí predisponen el control o prevención de la hipertensión arterial, debido a que el buen control, la buena alimentación y la prevención del estrés y el sedentarismo, son la base para tener una vida saludable.

\section{CONCLUSIONES}

1. Con respecto a las características de la población estudiada encontramos que:

- El género femenino fue el más prevalente en la hipertensión arterial de estadio 1, y en la hipertensión arterial de estadio de estadio 2 fue el género masculino.

- En cuanto a la edad los que presentaron hipertensión arterial estadio 2 pertenecían a las edades de 46 a 56 años; asimismo, los que tenían hipertensión arterial estadio 1 pertenecían a las edades de 35 a 45 años, al igual de los mayores de 67 años, así como también a las edades de 46 a 56 años.

- En cuanto a los antecedentes familiares, los que presentaron pre-hipertensión e hipertensión estadio 1 y 2 , manifestaron tener como antecedente familiar a la madre y al padre.

- Sobre el nivel de conocimientos de la la presencia de la hipertensión arterial, los que pre- 
sentaron hipertensión arterial estadio 1 y 2, sí tenían conocimiento sobre la presencia de la hipertensión arterial y los que presentaron prehipertensión, no sabían que presentaban dicha enfermedad.

2. Con respecto a la frecuencia de la hipertensión arterial, se encontró que 44 adultos mayores de 35 años presentaron pre-hipertensión; asimismo, 23 adultos mayores de 35 años presentaron hipertensión estadio 1 y 2 , solo 56 adultos mayores de 35 años mostraron presión arterial normal.

3. Los resultados estadísticos de la relación entre el nivel de conocimientos con la hipertensión arte- rial muestran que existe relación positiva muy débil, por lo que se concluye rechazar la hipótesis de investigación a favor de la nula.

4. Según los resultados estadísticos de la relación entre el nivel de prácticas de autocuidado con la hipertensión arterial, muestran que existe relación positiva considerable, por lo que se concluye rechazar la hipótesis nula a favor de la hipótesis de investigación. Los resultados estadísticos de la relación entre el nivel de conocimientos y prácticas de autocuidado con la hipertensión arterial muestran que existe una relación positiva débil. Por lo que se concluye en rechazar la hipótesis nula a favor de la hipótesis de investigación.

\section{REFERENCIAS BIBLIOGRÁFICAS}

1. Cayetano, H. U. (4 de febrero de 2004). Temas de salud y bienestar. El comercio, Discovery Health channel, págs. 102 - 104.

2. Diehl, H., \& Ludington, A. (2003). Vida dinámica. Buenos Aires, Argentina: Asociación Casa Editora Sudamerica.

3. Escott, S. S., \& Mahan, K. (2009). Krause Dietoterapia. Barcelona, España: El sevier Masson.

4. Jenkins, D. (2005). Mejoremos la salud a todas las edades, Un manual para el cambio de comportamiento. Washington: Organización Panamericana de la Salud.

5. OMS, (Organización Mundial de la Salud), (28 de abril de 2011). Neurociencias. Obtenido de http://www.neurodifusion.org/noticias/saludmedicina/63-noticias-salud-medicina/9508segun-la-oms-unos-52-millones-de-personaspodrian-morir-de-enfermedades-cronicas-notransmisibles-para-2030.html.

6. Perez, C. D., Cordiés, J. L., Vásquez, V. A., \& Serrano, V. C. (2004). Hipertensión arterial, guía para la atención médica. Cuba: Republica de Cuba, Ministerio de Salud Pública.

7. Rivera, A. L. (diciembre de 2006). Revista de Ssalud Publica. Obtenido de Capacidad de Agencia de Autocuidado en Personas con Hipertension Arterial hospitalizados en una
Clinica de Bogotà, Colombia. Recuperado, http:// www.scielo.unal.edu.co/scielo.php?pid=S012400642006000300009\&script=sci_arttext.

8. Rojas, A., \& Rojas, L. (abril de 1996). Revista peruana de cardiología. Recuperado, de prevalencia de la hipertensión arterial: http:// sisbib.unmsm.edu.pe/bvrevistas/cardiologia/ v22_n1/harterial.htm.

9. Saldarriaga, S. L. (2007). Valoración de conocimientos de hipertensión arterial y factores de riesgo. Nure Investigación , 4 - 6.

10. Sardaña, J. (2000). “Digame doctor”, La hipertensión. México: Editorial Diana S.

11. Smeltzer, S. C. \& Bare, B. G. (2005). Enfermeria medicoquirúrgica. España: McGraw-Hill Interamericana.

12. Torreblanca Roldán, M.C. (1997). Efecto de una intervención educativa en la calidad de vida del paciente hipertenso abril a mayo año 2004. Caplotan México.

13. Velázquez, B. (13 de mayo de 2011). Sociedad peruana de hipertensión arterial. Recuperado, de http://www.htaperu.pe/index.php?option=com content \&view $=$ article \&id=123:lo-que-debesaber\&catid=48:articuloas\&Itemid $=169$.

14. White, E. G. (2007). El ministerio de curaciòn. Argentina: Casa Editora Sudamericana. 